\title{
On the application of the Exp-function method to nonlinear differential-difference equations
}

\section{İsmail Aslan}

Department of Mathematics, Izmir Institute of Technology, Urla, Izmir 35430, Turkey

\section{A R T I C L E I N F O}

\section{Keywords:}

Exp-function method

Langmuir lattice

Relativistic Toda lattice system

\section{A B S T R A C T}

When applying the Exp-function method to nonlinear-differential difference equations, Bekir (2010) [1] reported incorrect results.

(c) 2012 Elsevier Inc. All rights reserved.

Recently, Bekir [1] investigated the Langmuir chains equation

$$
\frac{d u_{n}}{d t}-u_{n}\left(u_{n+1}-u_{n-1}\right)=0
$$

based on the ansatz

$$
u_{n}\left(\xi_{n}\right)=\frac{a_{1} \exp \left(\xi_{n}\right)+a_{0}+a_{-1} \exp \left(-\xi_{n}\right)}{\exp \left(\xi_{n}\right)+b_{0}+b_{-1} \exp \left(-\xi_{n}\right)}, \quad \xi_{n}=d n+c t+c_{0},
$$

and claimed that Eq. (1) admits the solutions

$$
\begin{aligned}
& u_{n}\left(\xi_{n}\right)=\frac{\frac{2 c}{e^{2 d}-e^{-2 d}} \exp \left(\xi_{n}\right)+a_{-1} \exp \left(-\xi_{n}\right)}{\exp \left(\xi_{n}\right)}, \quad \xi_{n}=d n+c t+c_{0}, \\
& u_{n}(t)=c \operatorname{csch}(2 d)+\cosh \left(2\left(d n+c t+c_{0}\right)\right)-\sinh \left(2\left(d n+c t+c_{0}\right)\right) .
\end{aligned}
$$

However, the direct substitution of the expression (3) into (1) yields

$$
e^{-2\left(d+2 d n+2 c t+2 c_{0}\right)}\left(e^{4 d}-1\right) a_{-1}^{2},
$$

which is not zero in the general case. Similary, it can be shown that (4) cannot be a solution of Eq. (1).

In fact, using the ansatz (2) in a straightforward manner, one can show that Eq. (1) admits the solution

$$
u_{n}(t)=\frac{c}{2} \operatorname{csch}(d)+\frac{2 c b_{0} \sinh (d) \exp \left(d n+c t+c_{0}\right)}{2(1+\cosh (d)) \exp \left(2\left(d n+c t+c_{0}\right)\right)+2 b_{0}(1+\cosh (d)) \exp \left(d n+c t+c_{0}\right)+b_{0}^{2}},
$$

where $b_{0}, d, c$, and $c_{0}$ are arbitrary parameters.

The same author also considered the relativistic Toda lattice system

$$
\frac{d u_{n}}{d t}-\left(1+\alpha u_{n}\right)\left(v_{n}-v_{n-1}\right)=0, \quad \frac{d v_{n}}{d t}-v_{n}\left(u_{n+1}-u_{n}+\alpha v_{n+1}-\alpha v_{n-1}\right)=0
$$

\footnotetext{
E-mail address: ismailaslan@iyte.edu.tr
} 
Then, via the transformation $v_{n}=(-1 / \alpha) u_{n}-1 / \alpha^{2}$, the author reduced the system (7) into the single equation

$$
\frac{d u_{n}}{d t}-\left(\frac{1}{\alpha}+u_{n}\right)\left(u_{n-1}-u_{n}\right)=0
$$

and claimed that Eq. (8) admits the solutions

$$
\begin{aligned}
& u_{n}(t)=\frac{\frac{1-e^{-d}+c \alpha e^{-d}}{\alpha\left(e^{-d}-1\right)} \exp \left(\xi_{n}\right)+\frac{\left(e^{d}-1+c \alpha d^{d}\right) b_{0}}{1-e^{d}}}{\exp \left(\xi_{n}\right)+b_{0}}, \quad \xi_{n}=d n+c t+c_{0}, \\
& u_{n}(t)=-c \operatorname{coth}(d)+\frac{1}{\alpha}\left(c \tanh \left(d n+c t+c_{0}\right)-\operatorname{sech}\left(d n+c t+c_{0}\right)\right), \\
& u_{n}(t)=\left(c-\frac{1}{\alpha}\right)-c(\operatorname{coth}(d)+\operatorname{csch}(\mathrm{d}))-\frac{c}{2}\left(\operatorname{csch}\left(d n+c t+c_{0}\right)+\operatorname{coth}\left(d n+c t+c_{0}\right)\right) .
\end{aligned}
$$

However, the direct substitution of the expression (9) into (8) yields

$$
-\frac{e^{c t+c_{0}}(\alpha-1)\left(e^{d(n-1)}+e^{d(n+1)}(1+c \alpha)-e^{d n}(2+c \alpha)\right)\left(1-(1+c) \alpha+e^{d}\left(\alpha-1+c \alpha^{2}\right)\right) b_{0}^{2}}{\left(e^{d}-1\right)^{2} \alpha^{2}\left(e^{d(n-1)+c t+c_{0}}+b_{0}\right)\left(e^{d n+c t+c_{0}}+b_{0}\right)^{2}},
$$

which is not zero in the general case. Similarly, one can show that the expressions (10) and (11) cannot be solutions of Eq. (8). In fact, based on the ansatz (2), one can show that Eq. (8) admits the solution

$$
u_{n}^{\mp}(t)=\frac{c}{1-\exp (d)}-\frac{1}{\alpha}-\frac{c\left(2 b_{-1}+\left(b_{0} \mp \sqrt{b_{0}^{2}-4 b_{-1}}\right) \exp \left(d n+c t+c_{0}\right)\right)}{2\left(\exp \left(2\left(d n+c t+c_{0}\right)\right)+b_{-1}+b_{0} \exp \left(d n+c t+c_{0}\right)\right)},
$$

where $b_{0}, b_{-1}, d, c$, and $c_{0}$ are arbitrary parameters.

As a result, we can conclude that Bekir made some errors in the application of the Exp-function method to nonlinear-differential difference equations.

\section{Reference}

[1] A. Bekir, Application of the Exp-function method for nonlinear differential-difference equations, Appl. Math. Comput. 215 (2010) $4049-4053$. 